\title{
Wide Field and Deep Focus imaging in Photomicrography \\ Optical and Software-Based Techniques
}

\author{
Jörg Piper \\ Clinic Meduna, Bad Bertrich, Germany \\ jpu.mp@t-online.de
}

\section{Introduction:}

In photomicrography, two fundamental problems often arise in practice: The higher the magnification and the higher the thickness or three dimensional extension of the specimen, the lower is the depth of focus which can result in parts of the specimen being indistinct. The larger the area of the specimen, the lower is the resolution in images showing the specimen in a total view. Additional problems will occur, when the lowest magnification of the respective microscope is not low enough for inclusion of the specimen's total size so that only parts of the specimen will be visible in photo micrographic images. Table 1 presents the focal depth and lateral resolution of microscopic objectives with regard to their magnification and numerical aperture (modified from [4] and [7]).

In this article, several solutions for these problems are described and discussed, based on optical components and specific software tools for computer-based image processing.

\section{Materials and Methods:}

The various techniques for wide field and deep focal imaging are demonstrated on examination of four different specimens (Fig. 1 and 2): An histological section of a stained mouse embryo (total length: $24 \mathrm{~mm}$ ), a stained section of the human eye (total length: 26 $\mathrm{mm}$ ), a wing of a butterfly (total length: $22 \mathrm{~mm}$ ) and a small starfish (diameter: $55 \mathrm{~mm}$ ), prepared in total.

The cover slip preparations were examined in transmitted light, using a common laboratory microscope (Leitz/Leica Dialux), the starfish was observed by a stereo microscope in epi-illumination.

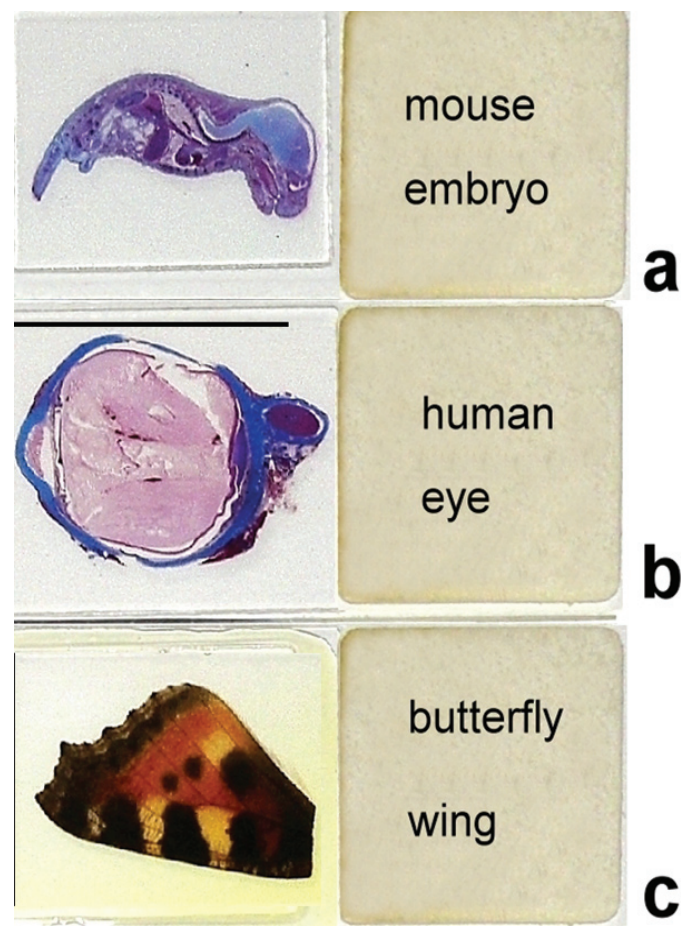

Fig. 1: Cover slip preparations of large-area specimen (further explanations in the text), a: mouse embryo, b: human eye, c: butterfly wing
The laboratory microscope was equipped with an Olympus $\mathrm{Ca}$ media C-7070 digital camera, the stereo microscope with a digital Canon Powershot A 95 compact camera. Macro images were taken by two different digital compact cameras (Casio Exilim EX-Z 110, 6 megapixels and a Sony Cybershot DSC-W 90 ,8 megapixels), and also by a digital mirror reflex camera (Canon EOS 350 D, APSchip, 8 megapix- els), equipped with an high-end macro lens (Leica Macro-Elmarit-

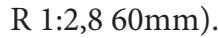

Computer-based, wide field images were composed with the help of stitching software ("panorama"-software); in this respect, AutoStitch [1] and PhotoStitch [2] lead to the best results. Three dimensional deep focus reconstructions were created by several stacking software (Combine Z 5 [5], Picolay [3], Helicon Focus [6]).

\section{Solutions and results in wide field imaging:}

When a large-area specimen has to be photographed, common digital cameras can be used in macro mode. According to several tests, chips containing 6 or 8 megapixels are suitable for this task. For physical reasons digital compact cameras equipped with small chips lead to an enlarged focal depth in close-up. Thus, good results can be achieved by freehand photography. Fig. 3 shows two freehand images of the mouse embryo both

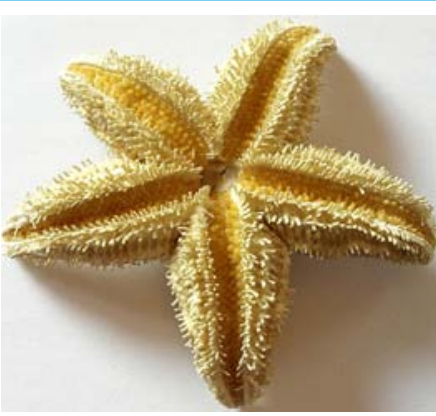

Fig. 2: Starfish, prepared in total, diameter: $55 \mathrm{~mm}$ (further explanations in the text)

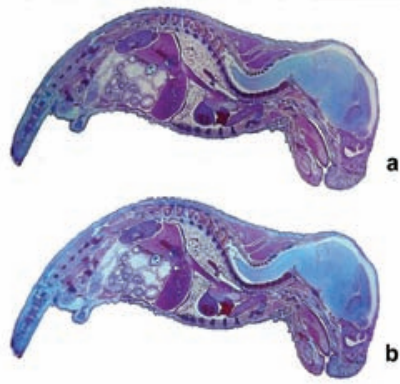

Fig. 3: Mouse embryo photographed with digital compact cameras in macro 110 (6 megapixels), b: Sony Cybershot DSC-W 90 (8 megapixels) mode, a: Casio Exilim EX-Z

taken with the digital compact cameras (Casio Exilim EX-Z and Sony Cybershot DSC-W90). The slide was fixed on a clear and clean windowpane and illuminated by skylight. The Casio camera leads to a higher planarity, the Sony camera is characterized by a higher local resolution but a lower peripheral sharpness. Nevertheless, these cameras seem both to be suitable for simple and easy documentations of large-area specimens.

Table 1: Typical values for depth of field and lateral resolution in light microscopy (modified from [4] and [7])

\begin{tabular}{|c|c|c|c|}
\hline $\begin{array}{c}\text { Magnification } \\
\text { of objectives }\end{array}$ & $\begin{array}{c}\text { Numerical } \\
\text { Aperture }\end{array}$ & $\begin{array}{c}\text { Depth of Field } \\
\text { (micrometers) }\end{array}$ & $\begin{array}{c}\text { Lateral } \\
\text { resolution } \\
\text { (micrometers) }\end{array}$ \\
\hline $4 \mathrm{x}$ & 0.10 & 55.5 & 4.5 \\
\hline $10 \mathrm{x}$ & 0.25 & 8.5 & 1.5 \\
\hline $20 \mathrm{x}$ & 0.40 & 5.8 & 0.9 \\
\hline $40 \mathrm{x}$ & 0.65 & 1.0 & 0.5 \\
\hline $80 \mathrm{x}$ & 0.85 & 0.4 & 0.4 \\
\hline $100 \mathrm{x}$ & 0.95 & 0.19 & 0.35 \\
\hline
\end{tabular}

Further improvements of the image quality were achievable when the digital mirror reflex camera was used together with the high end macro lens. When specimens are taken in transmitted light, equipment for reproductions of diapositives can be helpful for preparing the slide. Duplicating adapter suitable for this purpose can be bolted within the macro lens instead of a filter. Fig. 4 demonstrates the mouse embryo from fig. 1 and 3 , taken with the Canon EOS 20 D camera, equipped with the Leica macro lens and 


\section{COLLEGE OF MICROSCOPY}

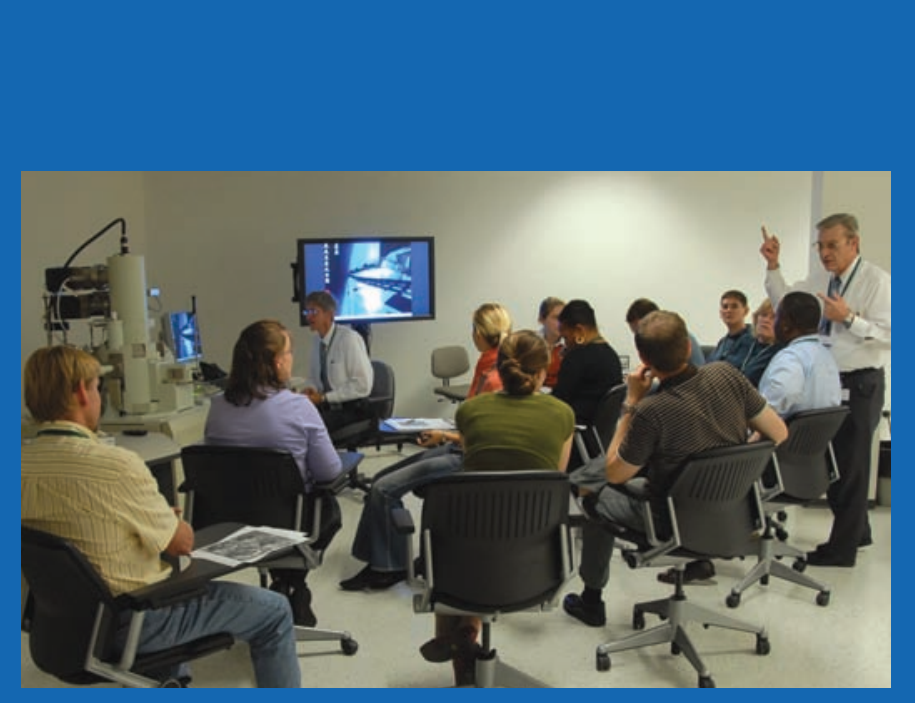

\section{Scanning Electron Microscopy Course March 31-April 4, and October 6-10, 2008}

The College of Microscopy's Scanning Electron Microscopy (SEM) course emphasizes hands-on learning. Using five SEM and EMA instruments, students have the opportunity to study their own samples, or test samples provided by our staff, under the direction of McCrone scientists with over 90 years of combined SEM/EDS/WDS experience. During the course, students will learn through lecture, demonstration, and handson participation how to setup and operate SEM and EDS instruments, including low-vacuum and field-emission models.

\section{LEARN FROM EXPERIENCE... LEARN FROMTHE EXPERTS}

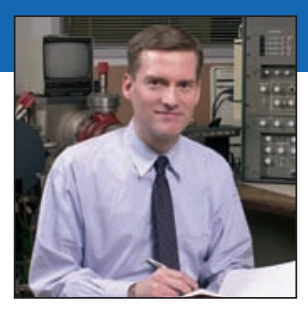

Kent has been with McCrone Associates since 1990. As Vice President, Director of Instrumental Analysis, his responsibilities include sample analysis, along with quality control and advanced data analysis. Areas of interest are WDS and surface analysis. Kent specializes in surface analytical techniques using $\mathrm{x}$-ray photoelectron spectroscopy, Auger spectroscopy, secondary ion mass spectrometry, and electron microprobe analysis.

Joe has been with McCrone Associates since 1986. As a senior research scientist in the electron optics group, he specializes in particle analysis, polymer imaging, electronics, artist pigments, medical devices, and general industrial problem solving, using SEM, EDS and WDS analysis. Joe is responsible for identification of inorganic and organic particles and thin films and a consultant in ultramicroanalysis and microscopy for clients from government and from the pharmaceutical, electronics, paint, automotive, packaging, and metals industries.
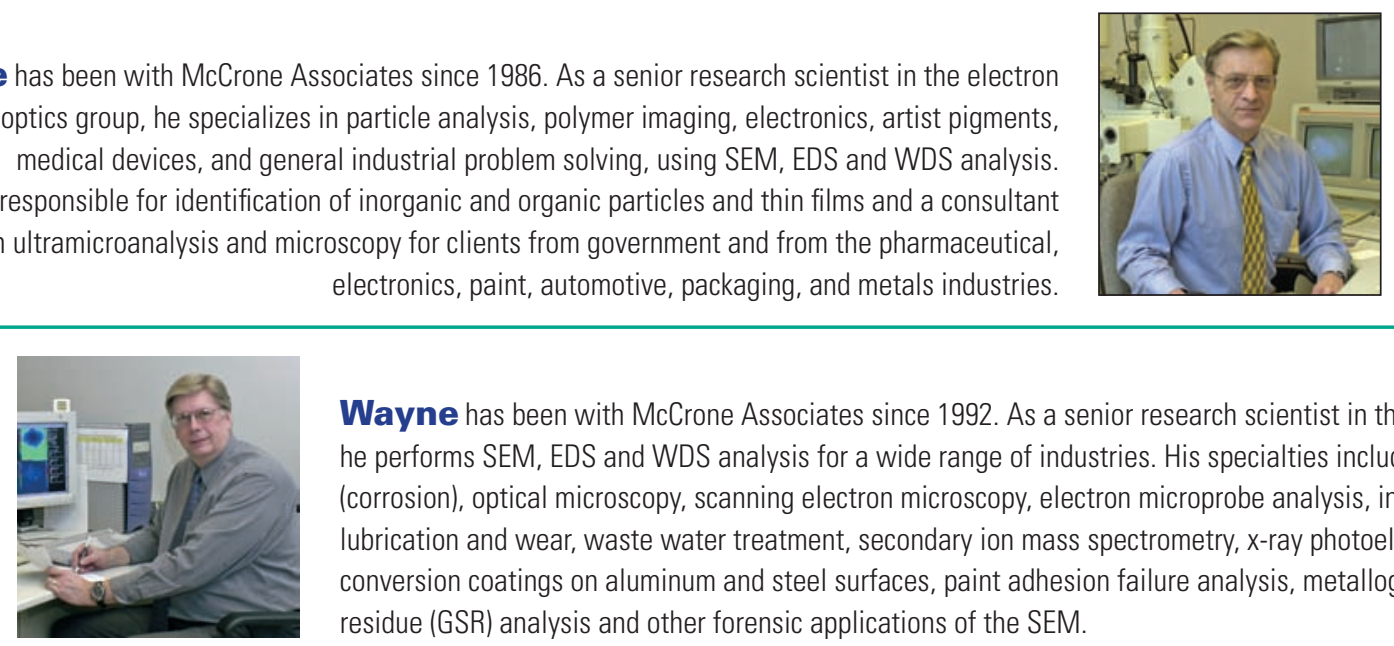

Wayne has been with McCrone Associates since 1992. As a senior research scientist in the electron optics group, he performs SEM, EDS and WDS analysis for a wide range of industries. His specialties include: electrochemistry (corrosion), optical microscopy, scanning electron microscopy, electron microprobe analysis, infrared spectroscopy, lubrication and wear, waste water treatment, secondary ion mass spectrometry, $x$-ray photoelectron spectroscopy, conversion coatings on aluminum and steel surfaces, paint adhesion failure analysis, metallography, gunshot residue (GSR) analysis and other forensic applications of the SEM.

Craig specializes in $\mathrm{x}$-ray microanalysis of particles using energy and wavelength dispersive spectrometry methods with the scanning electron microscope and electron microprobe. He has extensive and diverse analytical capabilities developed with extensive petrologic and mineralogic research. These include, among others, optical microscopy, x-ray diffraction analysis, electron microscopy, $\mathrm{x}$-ray spectrometry.

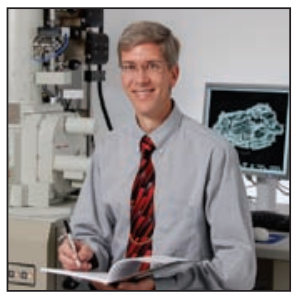




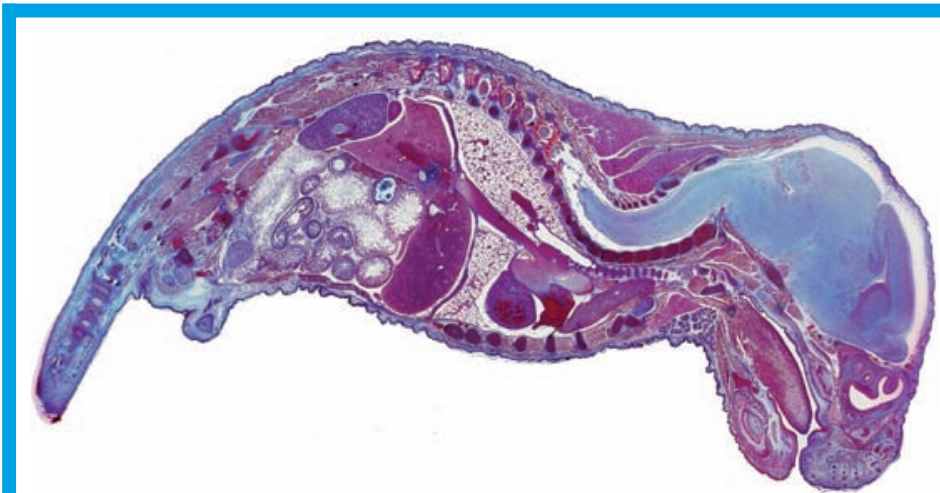

Fig. 4: Mouse embryo photographed with a Canon EOS 350 D mirror reflex camera, Leica macro lens and Hama duplicating adapter.

a duplicating adapter. It is evident that the macro lens and the high resolution chip of the mirror reflex camera lead to improved results when compared with digital consumer compact cameras. Sharpness, contrast, and planarity are optimized; the image quality seems to be comparable with stereomicroscopic results.

Using a light microscope, full size photos can be produced in large-area specimens by higher magnifying lenses when composite images are created with the help of specific stitching software. To achieve this, a sequence of various single images was taken, each showing a different part of the specimen. In this sequence, adjoining images must overlap in their marginal regions so that congruent structures in the marginal zones of these images can be detected by the software. Based on this, all single images can be arranged within a matrix and combined with each other so that a high resolution full size image of the specimen is reconstructed.

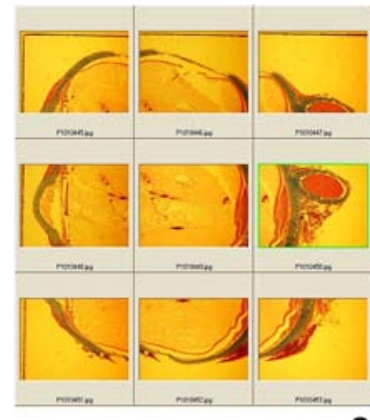

a

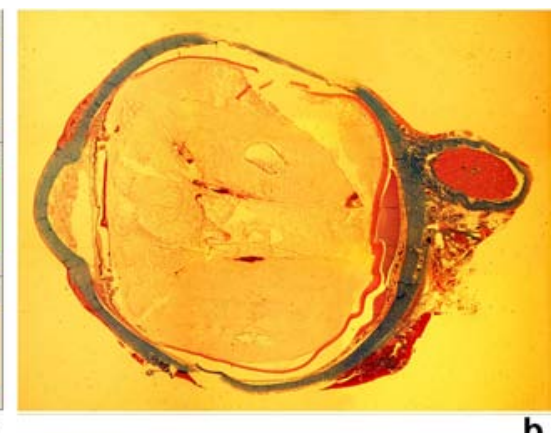

b
Fig. 5: Human eye, computer-based large-area reconstruction, bright field, plane objective 1,0x, ocular $5 x$ (software: PhotoStitch) ; a: matrix consisting of nine single images $b$ : resulting composed image

Fig. 5 shows the section of a human eye as an example for a software-based reconstructed image of a large-area specimen. In this case PhotoStitch was used as software. Intentionally, the specimen was illuminated in low brightness, so that the equalization of brightness within the yellowish background achievable by image processing is clearly documented. According to our own experiences, PhotoStitch (Canon utility) and AutoStitch (freeware) can be successfully used for these reconstructions in all fields of light microscopy.

\section{Solutions and results in deep focus imaging}

When details of three-dimensional specimens have to be documented in high magnification images, the depth of focus can be enhanced when the image is taken with a low magnifying lens; the lower the magnification, the higher is the focal depth. After this, small regions of interest can be clipped from the total image in a second step to achieve documents just showing interesting details.
Thus, small parts of the primary photo are used only for the final images. This technique is effective with lower resolution and lower contour sharpness when compared with images taken by higher magnifying lenses. On the other hand, these negative effects can be minimized by appropriate postprocessing techniques. The resulting final images can be improved with the help of software-based postprocessing procedures in several ways, e.g. reduction of noise, pixel interpolations, enhancements of contrast, contour sharpness and color saturation, modification of gradation etc.

All important parameters for image quality can be fundamentally improved when stacking software is used. By this technique, the details of interest can be taken with high magnifying lenses, so that the complete area of the primary photo can be used as final image. To achieve good results, a sequence of various single images must be taken in different focus zones. On this way, a complete vertical scan of the specimen is made; the principle of this procedure is the same as in radiological scanning techniques carried out in computer tomography and nuclear magnetic imaging. Next, all images of the scan are superimposed with each other with the help of the respective stacking software. In all images, only zones that are in focus are selected. The final three dimensional reconstruction resulting from this selective superposition shows a sharp and deep view of the specimen's detail that is free from any relevant regional indistinctness. With regard to focal depth, images composed by stacking software can be compared with results in scanning electron microscopy.
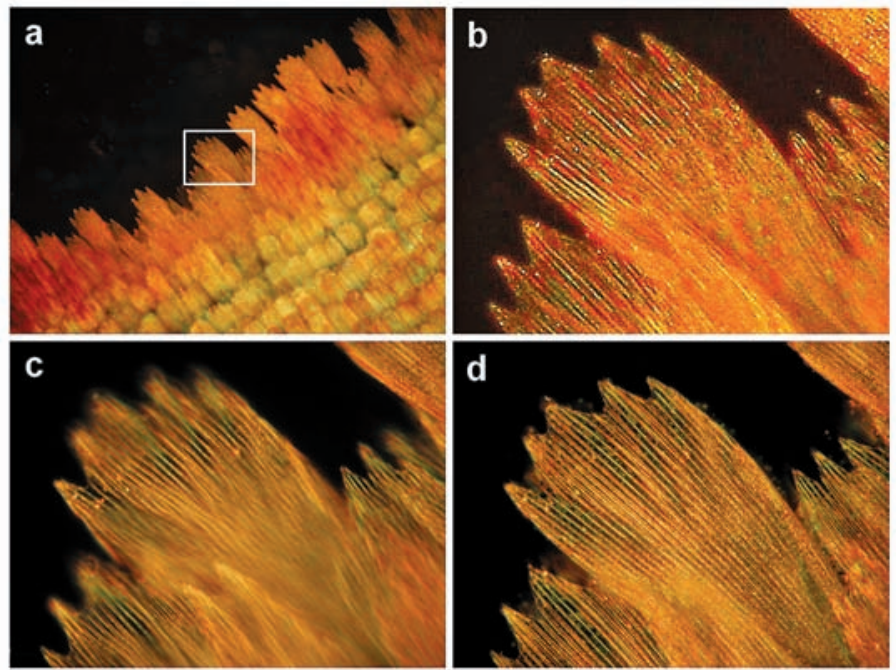

Fig. 6: Wing of a butterfly, dark field illumination: a: low magnified single image, plane objective 6,3x, ocular 10x, horizontal field width (HFW): 1,5 mm. The detail of interest (marginal scale) is marked by a white frame. b: section from fig. 7 a showing the interesting detail in high magnification, optimization of the resulting image quality by software-based postprocessing, HFW: 0,2 mm, c: high magnified image, plane objective 40x, ocular 10x, HFW: 0,2 mm. d: three dimensional reconstruction, based on 25 single images with different focal planes, plane objective 40x, eyepiece 10x, HFW: 0,2 mm (software: Combine Z 5)

Fig. 6 demonstrates the capabilities of the optical and softwarebased techniques described above. The butterfly wing, prepared in total, was successively photographed in dark field illumination using 6, 3-times and 40-times magnifying lenses. The final images resulting from the respective procedures show the same marginal scale of this wing in identical views. It can be seen that the 6, 3-times lens lead to a much higher depth of focus than a single shot taken with the 40-times lens. Moreover, it is evident, that the maximum image quality can be achieved by stacking techniques based on the higher 


\section{DIATOME} diamond knives

Development, Manufacturing, and Customer Service since 1970

What have we achieved in this period?

ultra $45^{\circ}$ the first diamond knife with an absolutely score-free, hydrophilic cutting edge.

semi the first diamond knife for alternating sectioning ultrathin/semithin.

cryo the diamond knife for sectioning at low temperature.

histo the first diamond knife for semithin sections for light microscopy.

ultra $35^{\circ}$ the diamond knife for optimized sectioning results in almost all applications.

STATIC LINE II the ionizer for eliminating electrostatic charging in ultramicrotomy.

cryo-P a cryo knife with a patented platform for section pick up.

cryo immuno the optimized cryo diamond knife for the Tokuyasu technique.

ultra sonic the oscillating diamond knife for room temperature sectioning.

cryotrim 45 and 25 optimizing trimming with diamond blades.

ultra AFM \& cryo AFM the first diamond knives for AFM at room and low temperatures.

cryo $25^{\circ}$ for sectioning frozen hydrated specimens.

\section{What services can we offer you?}

- Technical assistance in all fields of ultramicrotomy.

I Free sectioning tests for all types of samples.

- Make use of our many years of experience in perfecting our knives.

Custom knives, tools, and boats.

- Special purchase programs.

Workshops and training.

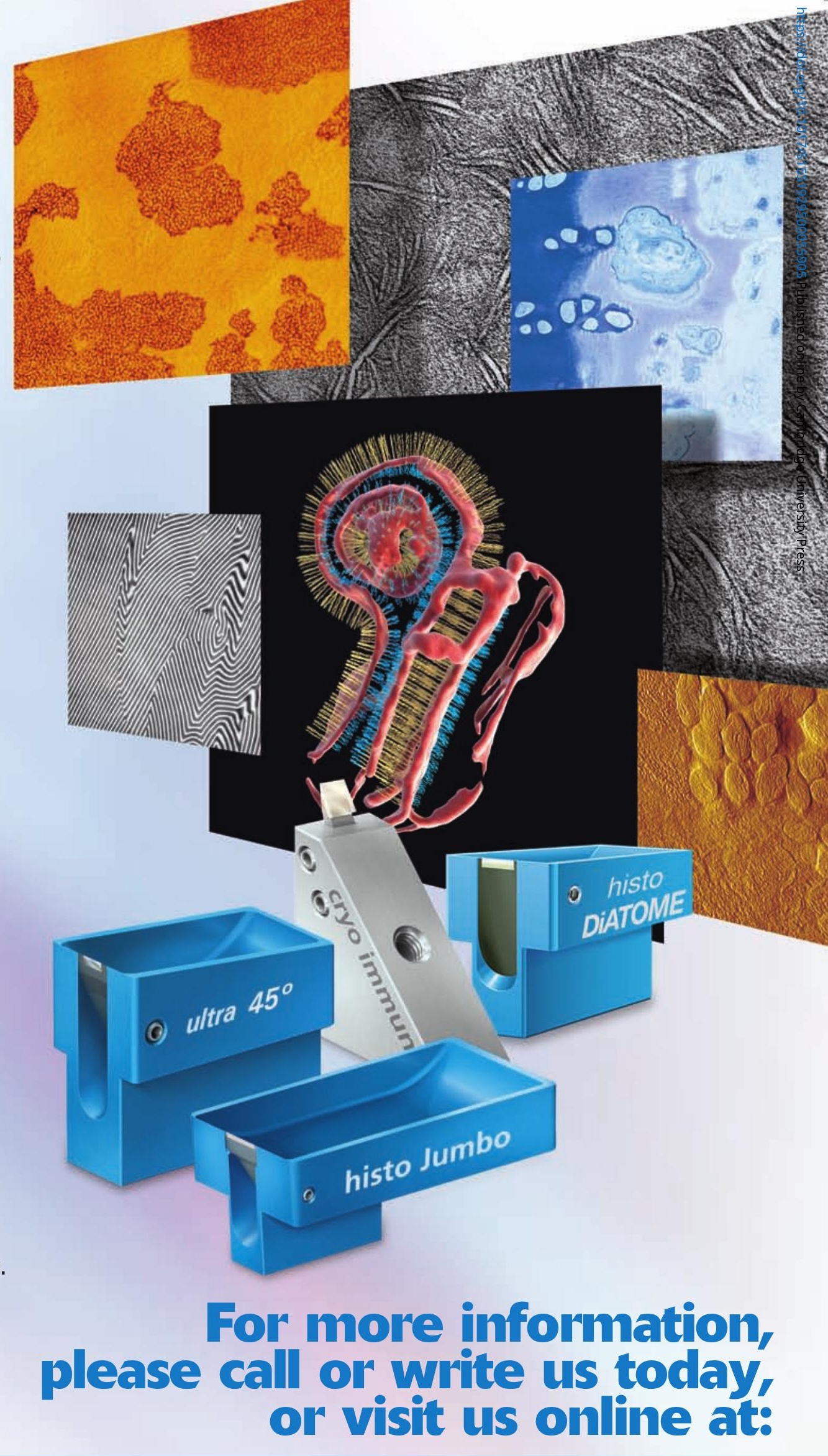

\section{www.emsdiasum.com}

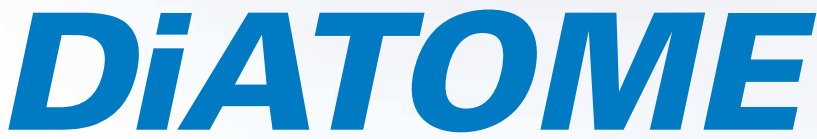

for all your sectioning requirements
P.O. Box $410 \bullet 1560$ Industry Rd.

Hatfield, Pa 19440

(215) 412-8390 • Toll Free: 1-(800) 523-5874

Fax: (215) 412-8450 or 8452

email:sgkcck@aol.com•stacie@ems-secure.com 


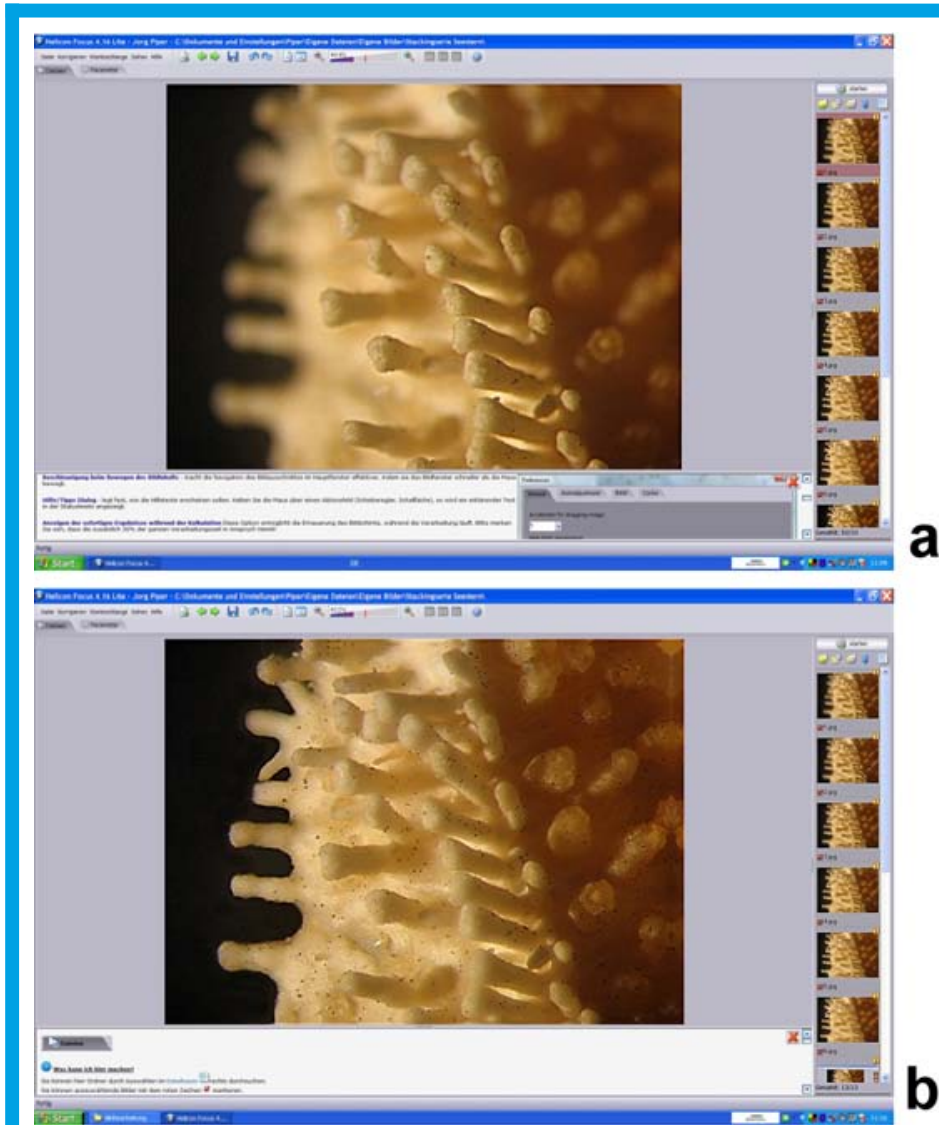

Fig. 7: Main screen in Helicon Focus, arm of a starfish, stereo microscope, epi-illumination, objective 2,0x, ocular 10x, HFW: $4 \mathrm{~mm}$, sequence of 7 single images with different focal planes (further explanations in the text): $a$ : initial situation, showing a representative single image, $b$ : final three-dimensional reconstruction

magnifying 40-times lens.

All stacking software tested lead to useful results so that it can be recommended for three dimensional reconstructions in light microscopy.

As an example for the principles of the computer-based workflow, fig. 7 shows some views of the main screen in the software Helicon Focus. Using a stereo microscope, various images were taken from an arm of the starfish shown in fig. 2. The complete sequence consisting of seven single images is arranged on the right; one exemplary single image and the final three dimensional reconstruction are situated on the left.

\section{Additional technical remarks:}

When wide field images of specimens are taken by digital cameras in macro mode, autofocus and automatic white balance software facilities work correctly in most cases. When a sequence of serial images is created for a software-based wide field composition it is very important for the resulting image quality that brightness and illumination are constant in all single images. Otherwise, inhomogeneities within the background will occur, because the stacking software mostly is not capable of equalizing all existing differences in brightness. According to our own experiences, AutoStitch seems to be more capable in equalizing such differences than PhotoStitch. On the other hand, in AutoStitch the processing time for rendering is about 10 times higher than using PhotoStitch.

All stacking software we evaluated work in a comparable and useful manner. Nevertheless, the software-specific algorithms for image processing differ from each other. Thus, in some cases, one particular software can lead to better results than the others. In monochromatic light, Helicon Focus, for the most part, works with superior precision, whereas Combine Z 5 and Picolay may not be capable in detecting sharp structures within monochrome images.

When serial images are taken with a stereo microscope the single images can be successively shifted in horizontal or vertical direction when the stage or the objective are moved up and down. In this situation, Combine $\mathrm{Z} 5$ is mostly capable in centering these stacks correctly, whereas the other tools may produce centering failures in the final reconstructions. Linear structures can be reconstructed in a maximum contour sharpness with the help of Combine $\mathrm{Z} 5$ in most cases. In automatic mode, just one preset is available in Helicon Focus, and three presets are offered by Combine Z 5 . In Picolay, five different presets are implemented so that five separate reconstructions are created from each image sequence when rendered automatically. Therefore, in some specimens Picolay can lead to superior results when reconstructions by the other tools may not be adequate. In some specimens, the resulting final sharpness can be enhanced further when two sequences of single images are separately superimposed and both resulting reconstructions are superimposed again in a second step (double stacking).

Apart from enhancements in the focal depth, stacking software can also be used for some other interesting tasks. For example, poor quality images caused by unsteadiness in the imaging system (camera shake) can be corrected, also when images of flat specimens with a low vertical depth are encountered, or when a stack of single images is superimposed consisting of several images with small differences in focus. In this way, the resulting global sharpness can be optimized in all microscopic applications. When a specimen is illuminated in very high contrast, e.g. in dark field, some regions can appear in maximized brightness, other zones in maximized darkness. In these cases, the homogeneity of brightness and contrast can be improved, when various single images are superimposed, despite being different in their exposure. Thus, ultrahigh ranges in brightness and contrast can be equalized. When a specimen is successively photographed in different illumination modes or illuminated by various light sources with different color temperatures or wave lengths, new and improved contrast effects can result by stacking the different images via a sandwich technique. Fig. 8 shows an example of a bicolor sandwich in phase contrast created by this technique. A native epithelial cell

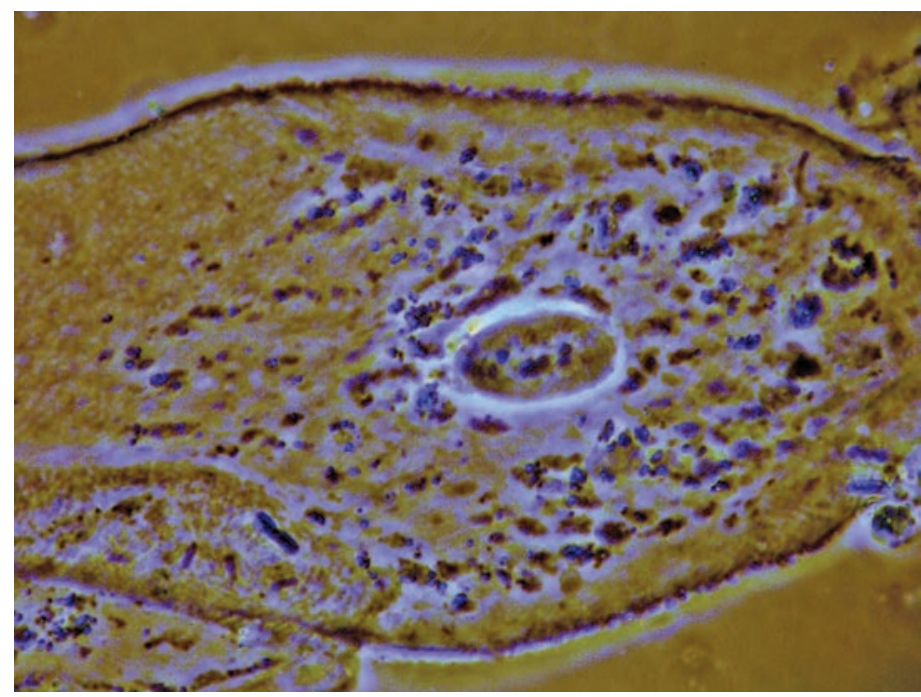

Fig. 8: Human epithelial cell from the oral mucosa, live-preparation, phase contrast, plane objective oil 100x, ocular 12,5x, HFW: 0,05 mm, superposition of 11 single images per sequence, double-stacking, bicolor sandwich technique (further explanations in the text) 
was taken in bulb and flash light illumination; both resulting images were superimposed using Combine Z 5. As the color temperatures are different, a new element of color contrast occurs whereby the fine structures, especially at the margin of the cell membrane, are accentuated because of the coincidence of red-yellow bulb light and blue flash light.

\section{Discussion:}

Wide field imaging is an interesting option especially for didactical purposes when specimens need to be photographed at life size to show a global view of their topography. When a transparent stained specimen is illuminated in transmitted light (bright field) large-area images can be taken by a digital camera used in macro mode. This technique is also suitable for non transparent specimens examined in epi-illumination. The lower the area of the sensor within the camera, the higher is the focal depth, but the lower is the maximum realizable resolution and the higher the potential noise level may be. Thus, it is dependent on the special characteristics of the specimen, which type of digital camera (compact or mirror reflex) will lead to better final results. However, computer-based compositions carried out with the help of stitching software, suitable also for common panorama views, are useful in all illumination modes implemented in light microscopy. Moreover, the resolution of composed images is much higher than in corresponding one-shot images, because higher magnifying lenses with a higher numerical aperture are used in this technique. On the other hand, much more processing time is necessary than taking one single image in macro mode.

In all fields of photomicrography, the achievable quality of images can be fundamentally improved with the help of stacking software. In principle, the depth of field can be maximized nearly infinitely so that final results are comparable with images in scanning electron microscopy. In many cases, specimens characterized by a large vertical dimension can only be photographed in a satisfying manner with the help of stacking techniques. Moreover, inaccuracy in focusing caused by physiological variability in the accommodation of the human eye can be avoided when the sharpness of fine details within the specimen is enhanced by stacking software. In special situations, additional contrast effects or an improved homogeneity in brightness and contrast can be achieved by sandwich techniques when several images are taken in different illumination or different exposure and superimposed with each other.

All software tools tested work in a very efficient manner. In most cases, the processing time for rendering is just a few minutes when modern computers are available. Therefore, these techniques can be easily integrated in each photographic microscopic workflow.

\section{References:}

[1] Brown, M., Lowe, D.G.:, Autostitch - a new dimension in automatic image stitching. Department for Computer Science, University of British Columbia. http://autostitch.net

[2] Canon Inc.: Original software and online photo services - photostitch. http:// www.canon.com/technology/canon_tech/explanation/software_dc.html

[3] Cyprionka, H.: Picolay - freeware for generating improved images from picture series, 2007. http:// www.picolay.de

[4] Hart, J.: 3D Microscopy and high-magnification macro-photography, Digital reconstruction and depth-of-field expansion. University of Colorado. http:// www.crystalcanyons.net/pages/TechNotes/3DMicroMacro.shtm, 2003

[5] Hedley, A.: CombineZ5. My software to combine pictures to increase depth of focus. http://www.hadleyweb.pwp.blueyonder.co.uk/index.htm, 2007

[6] Heliconsoft: Helicon Focus. http://www.heliconsoft.com/heliconfocus.html, 2007

[7] Leitz Wetzlar Comp.: Reproducing and illuminating optical components in light microscopy, manual, 1969 (in German)

\section{Find New \& Used Microscopes}

\section{Join over 120,000 members}

at

$\oplus$ Search over 1,250 microscope ads currently online

$\oplus$ Buy \& sell all major brands

$\oplus$ Free "wanted" ads for quick results

$\oplus$ Sell with no commission or final value fees

\section{Over 325 equipment categories} including:

Microscopes

Microscopes Accessories

Clinical Microscopes

Electron Microscopes

Image Analysis

Microtomes

Histology/Pathology

Semiconductor

\section{And Also. \\ Balances \\ Glassware \\ HPLC \\ GC}

Mass Specs

Pipettors

Mills/Grinders

Spectrophotometers

And More!

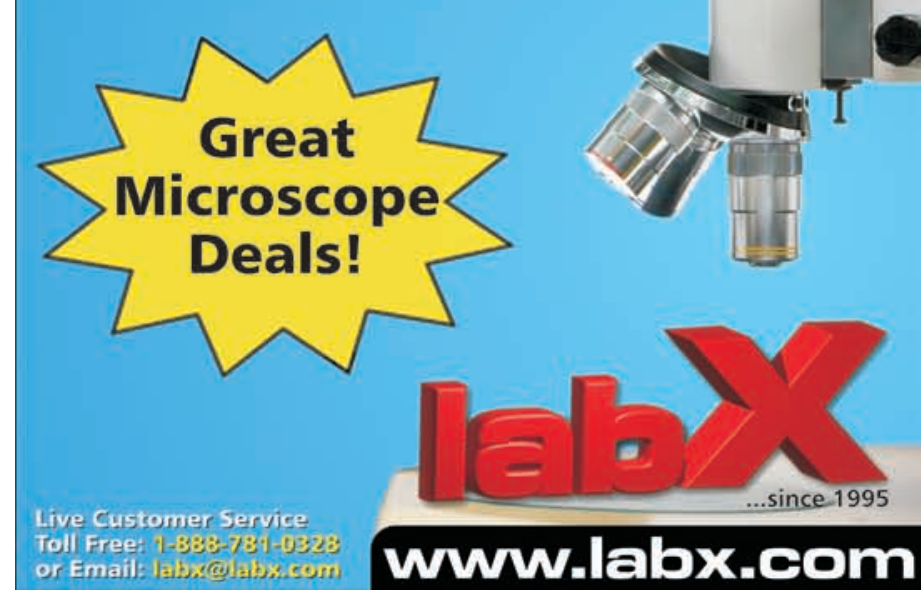

IICROSOOPY TOPAY March 2008 